\title{
Das Arbeitsverhältnis: Infragestellung des binären Modells?
}

\section{Einführung}

Die abhängige Beschäftigung stellt die Hauptarbeitsform des Wirtschaftssystems dar. ${ }^{1}$ Daran sind arbeitsrechtliche Schutzvorschriften geknüpft. Die in diesem Zusammenhang zu beantwortende Frage, wer als Arbeitnehmer klassifiziert werden kann, ist umstritten. Der Arbeitnehmerbegriff bildet bekanntermaßen die Grundlage des Arbeitsrechts. Trotz der Tatsache, dass einige Mitgliedstaten den Arbeitnehmerbegriff gesetzlich verankert haben und die Gerichte sich ausgiebig mit der Klassifizierungsfrage befasst haben, hat dieser Begriff im letzten Jahrzehnt immer mehr Fragen aufgeworfen. Grund dafür ist unter anderem das Aufkommen flexibler Arbeitsverhältnisse, worunter die atypischen ebenso wie die wirtschaftlich abhängigen Beschäftigten fallen. Auch das zunehmende Bedürfnis von Arbeitnehmern, sich als Selbständige zu etablieren um auf diesem Wege mehr Freiheit und ein höheres Einkommen zu erhalten, spielt dabei eine Rolle. Zudem ist die Grenze zwischen selbständiger Arbeit und unselbständiger Arbeit nicht mehr so deutlich wie sie noch vor einigen Jahren war. Das hat zur Folge, dass bestimmten Beschäftigten arbeitsrechtlichen Schutz vorenthalten wird. Deshalb hat die EG arbeitsrechtliche Richtlinien erlassen, die bezwecken sollen, dass die Rechtssicherheit der verschiedenen Arbeitnehmer verbessert wird. Dabei ist jedoch versucht worden, die Definition eines gemeinschaftsrechtlichen Arbeitnehmerbegriffes zu umgehen, indem in den Richtlinien bestimmt wird, dass der Arbeitnehmerbegriff national definiert wird. In diesem Artikel wird deshalb untersucht, ob diese Situation einer Veränderung bedarf. Überdies wird erörtert, ob man anstelle eines gemeinschaftsrechtlichen Arbeitnehmerbegriffs möglicherweise eine so genannte Zwischenkategorie auf gemeinschafts- bzw. nationaler Ebene einführen sollte. Zudem wird überlegt, ob auf europäischer Ebene ein Grundstock an Rechten für alle „abhängigen Beschäftigten“ realisiert werden sollte.

Zunächst wird der Arbeitnehmerbegriff im Allgemeinen erörtert (II.). Danach erfolgt zum einen die Darlegung des Arbeitnehmerbegriffs in der EG, wobei zunächst der gemeinschaftsrechtliche autonome Arbeitnehmerbegriff besprochen wird und zweitens die Definition des Arbeitnehmerbegriffs durch die Gemeinschaft (III.). Bevor wir zu unserer Schlussfolgerung kommen (V.), erfolgt noch eine Analyse der Argumente für und gegen einen durch die EG definierten Arbeitnehmerbegriff (IV.).

1 A. Perulli, Studie über wirtschaftlich abhängige Beschäftigungsverhältnisse/arbeitnehmerähnliche Selbstständige: rechtliche, soziale und wirtschaftliche Aspekte, S. 107 (http://ec.europa.eu/employment_social/news/2003/sep/parasubordination_report_de.pdf). Siehe ebenfalls Ch. Engels, Subordinate employees or self-employed workers, in: $\overline{\mathrm{R}}$. Blainpain, Comparative Labour Law and Industrial Market Economies, Kluwer 2007, S. 324. 


\section{Der Arbeitnehmerbegriff im Allgemeinen}

Erwerbstätige werden traditionell, d.h. nach dem binären Modell, in Selbständige und Arbeitnehmer eingeteilt. So wird der Anwendungsbereich des Arbeitsrechts abgegrenzt. ${ }^{2}$ Die Schutzvorkehrungen, die im Arbeitsrecht getroffen sind, gelten im Prinzip nicht für Selbständige, da diese im Allgemeinen keinen Bedarf an arbeitsrechtlichen Schutz haben. Sie bestimmen als ihr eigener Vorgesetzter ihre Arbeitsbedingungen selbst. Neben den eindeutig Selbständigen und Arbeitnehmern gibt es noch weniger klar einzustufende Beschäftigte, wobei andere Verträge als Arbeitsverträge geschlossen werden, welche in mancherlei Hinsicht dem Arbeitsvertrag ähneln. und wobei es ebenfalls um das erbringen einer Dienstleistung gegen Bezahlung geht. ${ }^{3}$ Es handelt sich hier um so genannte wirtschaftlich abhängige Beschäftigte bzw. arbeitnehmerähnliche Personen.

Die meisten Länder Europas besitzen keine (stichhaltig) gesetzlich verankerten Definitionen des Begriffs „Arbeitnehmer” oder auch des Begriffs „Arbeitsvertrag“ Somit sind in erster Linie die Gerichte für die Definition dieser Begriffe zuständig. Die Gesetzgebung der meisten Länder gibt allerdings gewisse feste Kriterien oder Indikatoren vor. Dies entspricht beispielsweise der Meinung der IAO, wonach die Mitglieder die Möglichkeit in Betracht ziehen sollten, ,, in ihren Rechtsvorschriften oder durch andere Mittel spezifische Indikatoren für das Vorliegen eines Arbeitsverhältnisses festzulegen ". ${ }^{4}$ In den meisten Ländern spielen der soziale Dialog und die Tarifverträge im Zusammenhang mit der Definition der vorstehend genannten Begriffe nur eine begrenzte Rolle.

Im Allgemeinen kann man den Arbeitnehmer jedoch definieren als eine Person, die Arbeit verrichtet und als Gegenleistung entlohnt wird. Sie steht in einem Verhältnis der Unterordnung zum Arbeitgeber. Neben den klar definierbaren Arbeitnehmern ${ }^{5}$ gibt es auch solche, die die Kriterien eines Arbeitnehmers nur teilweise erfüllen und dementsprechend nicht als Arbeitnehmer im eigentlichen Sinne klassifiziert werden können. Sie können aber auch nicht als selbständig betrachtet werden, da sie hauptsächlich nur für einen Auftraggeber arbeiten. Dass diese Personen, auch bekannt als arbeitnehmerähnliche Personen bzw. wirtschaftlich abhängige Beschäftigte, keinen Arbeitsvertrag haben, kann mehrere Gründe haben. Zum einen ist es möglich, dass der Auftraggeber sich nicht zu sehr an diese Person binden möchte und ihr deshalb keinen vollen arbeitsrechtlichen Schutz zukommen lassen will. Zum anderen besteht die Möglichkeit, dass diese Personen über die Einteilung ihrer Arbeit selbst bestimmen möchten oder dass sie

2 Siehe T. van Peijpe, Arbeitsvertrag und Scheinselbständigkeit in den Niederlanden, RdA 1998/4, S. 200.

3 Siehe auch Supiot, Beyond Employment - Changes in Work and the Future of Labour Law in Europe, OUP 2001, S. 22. Supiot unterscheidet prinzipiell drei verschiedene Arten der Erwerbstätigkeit. Zum einen die traditionellen Lohnempfänger die aufgrund eines Arbeitsvertrages arbeiten, wobei Unterordnung und/oder Weisung eine sehr wichtige Rolle spielt. Zweitens Erwerbstätigkeit aufgrund andere Verträge als Arbeitsverträge worin es gebt um das erbringen einer Dienstleistung mit als Gegenleistung Bezahlung. Zum Schluss unterscheidet er selbständige Unternehmer.

4 IAO Empfehlung 198 betreffend das Arbeitsverhältnis vom 31. Mai 2006.

5 D.h. solche Personen die klar als ein Arbeitnehmer im oben genannten Sinne klassifiziert werden können. 
auf einen finanziellen Vorteil abzielen, z.B. weil sie dann keine Sozialabgaben zu leisten brauchen. Neben den wirtschaftlich abhängigen Beschäftigten, sollten auch die atypischen Arbeitsverhältnisse genannt werden. Zu dieser Gruppe gehören z.B. befristet beschäftigte Arbeitnehmer, ${ }^{6}$ Teilzeitbeschäftigte, Heimarbeiter, Gelegenheitsarbeiter, freie Mitarbeiter oder Leiharbeitnehmer. ${ }^{78}$ Zudem gibt es auch Scheinselbständige, die Vollzeit arbeiten.

Es ist dem abhängig oder unabhängig Beschäftigten ebenso wie dem Arbeitgeber erlaubt, die Art ihres Vertrages zu bestimmen. So können beide Parteien sich für die Schließung eines Arbeitsvertrages entscheiden, oder aber auch z.B. einen Dienstvertrag miteinander vereinbaren. Nichtsdestotrotz ist es dem Richter überlassen, wie er das Arbeits- oder Dienstverhältnis interpretiert. Die Freiheit die diese Parteien haben, wird prinzipiell durch das Gesetz respektiert, solange das Gesetz unwiderlegbar die Art des Arbeits- oder Dienstverhältnisses bestimmt. ${ }^{9}$ Der Unterschied beider Vertragsformen liegt darin, dass der Arbeitnehmer, der aufgrund eines Arbeitsvertrages arbeitet, gegenüber dem Arbeitgeber weisungsgebunden ist. Bei einem Dienstvertrag hingegen fehlt im Allgemeinen die Weisungs- und Unterordnungsbefugnis des Auftraggebers.

Das Arbeitsverhältnis kreiert eine Beziehung zwischen dem Beschäftigten und dem Arbeitgeber. Der Arbeitsvertrag dient in vielen Mitgliedstaaten als Grundlage für ein solches Arbeitsverhältnis. Dafür werden verschiedene Kriterien benutzt, die dazu dienen, das Arbeitsverhältnis von anderen Beschäftigungsverhältnissen zu unterscheiden. Von einem Arbeitsverhältnis ist immer dann die Rede, wenn eine Person für den Arbeitgeber unter dessen Weisung arbeitet bzw. eine Dienstleistung erbringt und dafür als Gegenleistung eine Bezahlung erhält. Das heißt aber auch, dass Gesetze bestimmen können, dass bestimmte Personen(gruppen) nicht als Arbeitnehmer angesehen werden bzw. dass in bestimmten Situationen kein Arbeitsverhältnis vorliegt. Um zu bestimmen, ob ein Arbeitsverhältnis vorliegt, sollte nicht die Bezeichnung des Arbeitsvertrages im Mittelpunkt stehen, sondern die tatsächliche Ausführung des Vertrages. Zusätzlich verwenden die Mitgliedstaaten verschiedene Kriterien, die bestimmen, ob ein Arbeitsverhältnis vorliegt oder nicht.

Als Hauptkriterien in den Mitgliedstaaten der EG gelten die Unterordnung und/oder die Abhängigkeit des Arbeitnehmers von seinem Arbeitgeber. Zur Unterordnung ge-

6 Hier spielt Richtlinie 1999/70/EG eine Rolle. Richtlinie 1999/70/EG des Rates vom 28 Juni 1999 zu der EGB-UNICE-CEEP-Rahmenvereinbarung über befristete Arbeitsverträge, Amtsblatt Nr. L 175 vom 10.7.1999, S. 43-48. In dessen Anhang, Paragraph 3 ist ein befristet beschäftigter Arbeitnehmer ,eine Person mit einem direkt zwischen dem Arbeitgeber und dem Arbeitnehmer geschlossenen Arbeitsvertrag oder -verhältnis, dessen Ende durch objektive Bedingungen wie das Erreichen eines bestimmten Datums, die Erfüllung einer bestimmten Aufgabe oder das Eintreten eines bestimmten Ereignisses bestimmt wird".

7 Siehe für diesen Begriff Artikel 3(1)(c) der Richtlinie 2008/104/EG des Europäischen Parlaments und des Rates vom 19. November 2008 über Leiharbeit, Amtsblatt Nr. L 327 vom 5.12.2008, S. 9-14. Der Ausdruck Leiharbeitnehmer bezeichnet laut dem Text der Richtlinie: ,einen Arbeitnehmer, der mit einem Leiharbeitsunternehmen einen Arbeitsvertrag geschlossen hat oder ein Beschäftigungsverhältnis eingegangen ist, um einem entleihenden Unternehmen überlassen zu werden und dort unter dessen Aufsicht und Leitung vorübergehend zu arbeiten ".

8 Siehe ebenfalls für eine kurze Übersicht der atypischen Arbeitsverhältnisse: http://www.eurofound.europa.eu/docs/comparative/tn0801018s/tn0801018 s.pdf.

9 Engels (Fn. 1) S. 328. 
hören die folgenden Subkriterien: die organisatorische Fremdbestimmung; wirtschaftliche Indikatoren, wie z.B. wirtschaftliche Abhängigkeit und wirtschaftliche Unterordnung. ${ }^{10}$ Hinzu kommen noch eine Anzahl anderer Merkmale, die in den Mitgliedstaaten eine wichtige Rolle spielen: die Arbeit muss persönlich verrichtet werden; die Person ist dem Vertragspartner untergeordnet bzw. abhängig von ihm; die Arbeit wird durch den Vertragspartner beschafft; die Person bekommt die Arbeitsmaterialien durch den Vertragspartner bereit gestellt und die Arbeit wird zu einem vereinbarten Zeitpunkt erledigt. ${ }^{11}$

Es ist darauf hinzuweisen, dass verschiedene Interpretationen eine falsche Klassifizierung von Personen zur Folge haben können. Im Allgemeinen befindet der Arbeitgeber über die Klassifizierung, wenn er Personen beschäftigt bzw. mit ihnen einen Arbeitsoder Dienstleistungsvertrag schließt. Die Anzahl derer, die sich bewusst für die Selbständigkeit entschieden haben gegenüber denen, die sich (ungewollt) damit abfinden, kann nicht ermittelt werden. Es gibt einige - Kosten sparende - Gründe, weshalb ein Arbeitgeber eine Person als selbständig klassifiziert. So braucht der vermeintliche Arbeitgeber keine Steuern zu zahlen bzw. keine Sozialabgaben als auch Rentenabgaben für den Selbständigen zu leisten. Die Konsequenzen für den vermeintlich Selbständigen sind jedoch groß, da ihm z.B. bestimmte Rechte, auf die er als Arbeitnehmer Anspruch hätte, verwehrt werden. So fehlen ihm Rentenansprüche, ${ }^{12}$ womöglich Urlaubsgeld und Urlaubstage, eine anteilige Bezahlung der Sozialabgaben durch den Arbeitgeber und Bezahlung entsprechend dem Mindestlohn. Erfahren die Verwaltungsbehörden von der falschen Klassifizierung, haben sie die Befugnis, die fehlenden Steuer- und Sozialabgaben einzufordern. Zudem können sie dem vermeintlichen Auftraggeber ein Bußgeld auferlegen und eventuell auch noch Strafsanktionen verhängen. Der vermeintliche Selbständige kann die von ihm geleisteten Steuer- und Sozialabgaben zurückverlangen, sowie auch seine Rentenansprüche einfordern. Dem Arbeitgeber droht eine höhere Haftung für den Arbeitnehmer, die für Selbständige nicht gilt. Dadurch verliert das betref-

10 Siehe für eine Rechtsvergleichung R. Rebhahn, Der Arbeitnehmerbegriff in vergleichender Perspektive, RdA 3/2009, S. 154-175.

11 Gerade die unterschiedliche Auslegung der oben genannten Indikatoren kann - eventuell zu Problemen führen. Eines dieser Probleme ist die so genannte Scheinselbständigkeit. Diese unterschiedlichen Herangehensweisen bringen Diskrepanzen mit sich. So hat derjenige, der sich in einem unselbständigen Arbeitsverhältnis befindet, Anspruch auf bestimmten arbeitsund sozialrechtlichen Schutz. Er kann sich z.B. berufen auf maximale Arbeitszeiten sowie auch auf einen gesetzlichen Mindestlohn. Der Selbständige hingegen fällt - in den meisten Fällen - außerhalb des Geltungsbereichs des Arbeits- und Sozialrechts, gerade weil er sich eben nicht in einem unselbständigen Arbeitsverhältnis befindet und deshalb keinen Schutz vor, insbesondere Kündigungsschutz, z.B. Arbeitgebern, braucht.

12 Dieses ist nicht der Fall in den Niederlanden, da das Bezahlen einer Prämie und die Rente unabhängig voneinander sind. Es ist demnach auch zu einem späteren Zeitpunkt noch möglich zu beweisen, dass man ein Arbeitnehmer ist, und demnach Anspruch auf eine Rente hat, auch wenn bislang keine Prämie gezahlt worden ist. 
fende Unternehmen seinen Wettbewerbsvorteil, den es hatte, als der betreffende Arbeitnehmer noch als Selbständiger klassifiziert wurde. ${ }^{13}$

Im Prinzip können juristische Personen keine Arbeitnehmer sein. Nun stellt sich die Frage, inwieweit dies ebenfalls gilt für Ein-Personen Unternehmen, auch bekannt als own account workers. ${ }^{14}$ Diese Personen sind immer oder hauptsächlich abhängig von einem Auftraggeber. Wenn diese Person sich weiterhin nicht bemüht, andere Auftraggeber zu bekommen, für die sie Dienstleistungen erbringt, kann vermutet werden, dass diese Person mit einem Arbeitnehmer gleichgestellt werden kann. Nun gibt es Ein-Personen Unternehmen, die selbständig sein wollen, da sie möglicherweise keinen besonderen Schutz brauchen, aber auch weil sie eben die Vorteile des Selbständigseins genießen möchten. Für diese Personen ist es äußerst unangenehm, mit einem Arbeitnehmer gleichgestellt zu werden, denn gerade diese Situation wollten sie vermeiden.

Nachdem die Probleme und Komplikationen des Arbeitnehmerbegriffs erläutert worden sind, schauen wir uns zunächst verschiedene Lösungsvorschläge an, die am Ende des Beitrags analysiert werden.

\section{Der Arbeitnehmerbegriff in gemeinschaftsrechtlicher Perspektive}

Das Europäische Parlament ,,forderte eine Initiative zur Angleichung der einzelstaatlichen Arbeitnehmer-Definitionen im Interesse einer kohärenteren und effizienteren Anwendung des Gemeinschaftsrechts". Zudem forderte es die Mitgliedstaaten auf, die Durchführung der ILO-Empfehlung 198 betreffend das Arbeitsverhältnis zu fördern. Aus einigen Mitgliedstaaten kam sogar der Vorschlag, ,, die Empfehlung als Grundlage für eine Diskussion der Mitgliedstaaten und der Sozialpartner über die Frage zu nutzen, wie dem Problem der Scheinselbständigkeit auf europäischer Ebene besser begegnet werden kann". 15

Man sieht demnach, dass es Stimmen für einen gemeinschaftsrechtlichen Arbeitnehmerbegriff gibt, also einem Begriff, der nur in grenzüberschreitenden Situationen Anwendung findet. Zu überlegen ist jedoch auch, ob diese Einführung eines solchen gemeinschaftsrechtlichen Begriffs nicht auch innerstaatlich Geltung beanspruchen sollte.

Da der Arbeitnehmerbegriff nicht im EGV selber verankert ist, hat der EuGH im Hinblick auf die Freizügigkeit der Arbeitnehmer einen autonomen Arbeitnehmerbegriff entwickelt. Außerdem gibt es Richtlinien, die vereinzelte Teile des Arbeitsrechts harmonisieren. Jedoch der Arbeitnehmerbegriff, der in die Richtlinien aufgenommen ist,

13 Th. R. Bundy, Worker Misclassification: The Next Big Legal Concern?, Employee Relations Law Journal, Vol. 33, No. 3, winter 2007, S. 22. Siehe auch B. Grandi, Would Europe Benefit from the Adoption of a Comprehensive Definition of the Term 'Employee' Applicable in all Relevant Legislative Modes?, International Journal of Comparative Labour Law and Industrial Relations 2008, Vol. 24/4, S. 500-501.

14 Engels (Fn. 1) S. 331. Diese sind aufgrund der Zwölften Richtlinie 89/667/EWG des Rates vom 21. Dezember 1989 auf dem Gebiet des Gesellschaftsrechts betreffend Gesellschaften mit beschränkter Haftung mit einem einzigen Gesellschafter, Amtsblatt Nr. L 395 vom 30.12.1989, S. 40-42, in allen Mitgliedstaaten erlaubt.

15 Europäische Kommission, Ergebnis der öffentlichen Anhörung zum Grünbuch der Kommission, Ein moderneres Arbeitsrecht für die Herausforderungen des 21. Jahrhunderts, $\operatorname{KOM}(2007) 627$ endgültig, S. 8. 
wird nationalrechtlich definiert. Deshalb ist man bei Richtlinien auch immer davon ausgegangen, dass nur das nationale Arbeitnehmerkonzept in Betracht zu ziehen ist.

\section{Der gemeinschaftsrechtliche autonome Arbeitnehmerbegriff im Sinne des EGVs}

Artikel 39 EGV $^{16}$ gibt Arbeitnehmern - d.h. abhängig Beschäftigen - das Recht, Arbeit zu suchen so wie auch in einem anderen Mitgliedstaat zu arbeiten als dem, dem sie angehören. Dieses Recht können sie in gleicher Weise beanspruchen wie Arbeitnehmer in dem betreffenden Mitgliedstaat.

Der Hintergrund der Einführung der Freizügigkeit der Arbeitnehmer im EGV ist der, dass Arbeitnehmern aus Mitgliedstaaten mit einer hohen Arbeitslosigkeit die Möglichkeit gegeben werden sollte, um in einem anderen Mitgliedstaat Arbeit zu suchen und einer Beschäftigung nachzugehen. Diese Freizügigkeit hat sich als nicht ganz problemlos erwiesen. Deshalb hat der Europäische Gerichtshof in seiner Rechtsprechung versucht, mögliche Hindernisse, die der Freizügigkeit im Wege standen oder auch noch stehen, zu beseitigen. Das hat dazu geführt, dass seit dem Vertrag von Maastricht Arbeitnehmer als EU-Bürger Rechte genießen, die sie im Gast- oder Arbeitsland zur Geltung bringen können. ${ }^{17}$

Obwohl der Begriff „Arbeitnehmer“ in Artikel 39 EGV genannt wird, ist er nicht vertraglich definiert. Allerdings hat der EuGH eine weit auszulegende Arbeitnehmerdefinition entwickelt, anhand derer bestimmt werden kann, wer Arbeitnehmer im Sinne des EGV ist. Diese Definition kommt jedoch nur dann zur Geltung, wenn es sich um eine zwischenstaatliche Situation ${ }^{18}$ handelt. Dasselbe gilt ebenfalls für die anderen im EGV verankerten Freizügigkeiten.

In seinem Urteil Lawrie-Blum hat der Europäische Gerichtshof festgelegt, dass die Freizügigkeit der Arbeitnehmer eines der Grundprinzipien der Gemeinschaft ist. Demnach kann der Begriff des Arbeitnehmers im Sinne von Artikel 39 EGV (vorher Artikel 48) nicht je nach nationalem Recht unterschiedlich ausgelegt werden, sondern hat er

16 Artikel 39 EGV lautet:

,(1) Innerhalb der Gemeinschaft ist die Freizügigkeit der Arbeitnehmer gewährleistet.

(2) Sie umfasst die Abschaffung jeder auf der Staatsangehörigkeit beruhenden unterschiedlichen Behandlung der Arbeitnehmer der Mitgliedstaaten in Bezug auf Beschäftigung, Entlohnung und sonstige Arbeitsbedingungen.

(3) Sie gibt - vorbehaltlich der aus Gründen der öffentlichen Ordnung, Sicherheit und Gesundheit

gerechtfertigten Beschränkungen - den Arbeitnehmern das Recht,

a) sich um tatsächlich angebotene Stellen zu bewerben;

b) sich zu diesem Zweck im Hoheitsgebiet der Mitgliedstaaten frei zu bewegen;

c) sich in einem Mitgliedstaat aufzuhalten, um dort nach den für die Arbeitnehmer dieses

Staates geltenden Rechts- und Verwaltungsvorschriften eine Beschäftigung auszuüben;

d)nach Beendigung einer Beschäftigung im Hoheitsgebiet eines Mitgliedstaats unter

Bedingungen zu verbleiben, welche die Kommission in Durchführungsverordnungen festlegt.

(4) Dieser Artikel findet keine Anwendung auf die Beschäftigung in der öffentlichen Verwaltung. “.

17 C. Barnard, European Employment Law, OUP 2006, S. 171 und 172.

18 Im englischen auch cross-border element genannt. 
eine gemeinschaftsrechtliche Bedeutung. ${ }^{19}$ Das hat zur Folge, dass der gemeinschaftsrechtliche Begriff des Arbeitnehmers, da er den Anwendungsbereich dieser Grundfreiheit festlegt, weit auszulegen ist. Dieser Begriff ist anhand objektiver Kriterien zu definieren, die das Arbeitsverhältnis im Hinblick auf die Rechte und Pflichten der betroffenen Personen kennzeichnen. Das Urteil des EuGH lautete: „Das wesentliche Merkmal des Arbeitsverhältnisses besteht darin, dass jemand während einer bestimmten Zeit für einen anderen nach dessen Weisung Leistungen erbringt, für die er als Gegenleistung eine Vergütung erhält. " Die Studienreferendarin im vorliegenden Fall unterstand während der gesamten Dauer des Vorbereitungsdienstes der Weisung und der Aufsicht der Schule, der sie zugewiesen war, die ihr die erbringenden Leistungen und die Arbeitszeiten vorschrieb, deren Anweisungen sie auszuführen und an deren Vorschriften sie sich zu halten hatte. Für die erbrachten Dienstleistungen hat die Referendarin eine Gegenleistung erhalten. Somit waren die drei Kriterien für das bestehen eines Arbeitsverhältnisses erfüllt. ${ }^{20}$

Zusätzlich muss bei der Definition des Arbeitnehmers beurteilt werden, ob die ausgeübten Tätigkeiten als ,,tatsächliche und echte wirtschaftliche Tätigkeiten angesehen werden können "21 (im Sinne des Artikels 2 EGV). In Bettray hat der EuGH entschieden, dass die ausgeübten Tätigkeiten nur ein Mittel der Rehabilitation oder der Wiedereingliederung der Arbeitnehmer in das Arbeitsleben darstellen. Die entgeltliche Arbeit, die auf die körperlichen und geistigen Möglichkeiten des einzelnen zugeschnitten ist, soll den Betroffenen wieder ermöglichen, einer gewöhnliche Beschäftigung nachzugehen. Aus Raulin folgt, dass die Tätigkeiten auch nicht einen zu geringen Umfang haben, sich mithin nicht als völlig untergeordnet und unwesentlich darstellen dürfen. ${ }^{22}$ Allerdings können auch Arbeitnehmer, die in Teilzeit arbeiten, unter der autonomen Definition des Arbeitnehmers fallen. Das hat der EuGH in den Rechtssachen Levin und Kempf bestätigt. ${ }^{23}$ Auch die Personen, die Arbeit suchen, fallen unter dem autonomen Arbeitnehmerbegriff. ${ }^{24}$ Demzufolge muss es Arbeitnehmern erlaubt sein, sich mindestens drei

19 Siehe ebenfalls die Rechtssache Unger. Dort hat der Gerichtshof entschieden dass die Begriffe Arbeitnehmer und Tätigkeit im Lohn- und Gehaltsverhältnis nicht durch Verweisung auf die Rechtsvorschriften der Mitgliedstaaten definiert werden. Vielmehr haben sie eine gemeinschaftsrechtlichte Bedeutung, dessen Ziel es ist, dass die Mitgliedstaaten bestimmte Personengruppen nach belieben den Schutz des Vertrages entziehen zu können. Rechtssache 75-63 Urteil des Gerichtshofes vom 19. März 1964, Hoekstra (née Unger), Slg. 1964, S. 381.

20 Rechtssache 66/85 Urteil des Gerichtshofes vom 3. Juli 1986, Lawrie-Blum, Slg. 1986 S. 2121, Rdn. 16 bis 18. Siehe ebenfalls Rechtssache 53/81, Levin, Urteil vom 23. März 1982, Slg. 1982, S. 1035.

21 Rechtssache 344/87 Urteil des Gerichtshofes vom 31. Mai 1989, Bettray, Slg. 1989, S. 1621, Rdn. 17.

22 Rechtssache C-357/89 Urteil des Gerichtshofes vom 26. Februar 1992, Raulin, Slg. 1992, S. I-1027, Rdn. 10. Mit Hinweis auf Rechtssache 344/87 Urteil des Gerichtshofes vom 31. Mai 1989, Bettray, Slg. 1989, S. 1621, Rdn. 16.

23 Rechtssache 53/81 Urteil des Gerichtshofes vom 23. März 1982, Levin, Slg. 1982, S. 1035, Rdn. 17. Rechtssache 139/85 Urteil des Gerichtshofes vom 3. Juni 1986, Kempf, Slg. 1986, S. 1741, Rdn. 12.

24 Rechtssache C-85/96 Urteil des Gerichtshofes vom 12. Mai 1998, María Martínez Sala, Slg. 1998, S. I-2691, Rdn. 32. 
Monate lang nach einer passenden Arbeit in einem anderen Mitgliedstaat umzuschauen. ${ }^{25}$

Neben der umfassenden Rechtsprechung des EuGH in Bezug auf Arbeitnehmer hat der EuGH sich ebenfalls, wenn auch nur eher beiläufig, mit der Kategorie der „Selbständigen" beschäftigt. Der EuGH hat in Jany geurteilt, dass das wesentliche Merkmal eines Arbeitsverhältnisses darin besteht, dass jemand während einer bestimmten Zeit für einen anderen nach dessen Weisung Leistungen erbringt, für die er als Gegenleistung eine Vergütung erhält. Besteht kein Unterordnungsverhältnis, so ist diese Tätigkeit als eine selbständige Erwerbstätigkeit im Sinne des Artikels 43 EGV (vorher 52) zu qualifizieren. ${ }^{26}$ Diese selbständige Erwerbstätigkeit muss gemäß Artikel $43 \mathrm{EGV}$, wie auch der Arbeitnehmerbegriff gemäß Artikel $39 \mathrm{EGV}$, weit ausgelegt werden. ${ }^{27}$

\section{Rechtsprechung in Bezug auf den Arbeitnehmerbegriff in den EG-Richtlinien}

Die EG-Richtlinien, die als Ziel haben, den Arbeitsschutz zu sichern, haben verschiedene Geltungsbereiche in den Mitgliedstaaten. Es kommt schließlich darauf an, wen die Richtlinie für das Gemeinschaftsrecht als Arbeitnehmer ansieht. Z.B. heißt in Artikel 3 Absatz 1 c) der Richtlinie 2001/23/EG: ,, 'Arbeitnehmer' ist jede Person die in dem betreffenden Mitgliedstaat aufgrund der einzelstaatlichen Arbeitsrechts geschützt, ist ". Dieses folgt ebenfalls aus der Rechtssache Hoekstra wo der EUGH befunden hat, dass die Begriffsbedeutung „, Arbeitnehmer“ den Mitgliedstaaten überlassen ist. ${ }^{28}$ Ähnlich auch die Richtlinie 97/81/EG, die sagt, dass diese , [ ...] gilt für Teilzeitbeschäftigte, die nach den Rechtsvorschriften, Tarifverträgen oder Gepflogenheiten in dem jeweiligen Mitgliedstaat einen Arbeitsvertrag haben oder in einem Arbeitsverhältnis stehen".

Ein Beispiel das hier genannt werden kann, ist die Rechtssache Mikkelsen. In dieser Rechtssache ging es um Richtlinie 77/187/EEG und die Frage, inwieweit diese Richtlinie die Ansprüche der Arbeitnehmer beim Übergang eines Unternehmens. gewähr-

25 Eine Dauer von sechs Monaten, so wie in der Rechtssache Antonissen, ist kompatibel mit dem EGV. Rechtssache C-292/89 Urteil des Gerichtshofes vom 26. Februar 1991, Antonissen, Slg. 1991, S I-745, Rdn. 21. Sowie auch Rechtssache C-344/95 Urteil des Gerichtshofes vom 10. Juli 2003, Collins, Slg. 2004, S. I-2703.

26 Rechtssache C-268/99 Urteil des Gerichtshofes vom 20. November 2001, Jany, Slg. 2001, S. I-8615, Rdn. 34, 70 und 71. Siehe ebenfalls Rechtssache C-107/94 Urteil vom 27. Juni 1996, Asscher, Slg. 1996, I-3089, Rdn. 25 und 26.

27 Rechtssache C-257/99 Urteil des Gerichtshofes vom 27. September 2001, ex parte Julius Barkoci und Marcel Malik, Slg. 2001 Seite I-6557. Und Rechtssache C-55/94 Urteil des Gerichtshofes vom 30. November 1995, Gebhard, Slg. 1995 Seite I-4165, Rdn. 25, wo es heißt: „Der Begriff der Niederlassung im Sinne des Vertrages ist also ein sehr weiter Begriff, der die Möglichkeit für einen Gemeinschaftsangehörigen impliziert, in stabiler und kontinuierlicher Weise am Wirtschaftsleben eines anderen Mitgliedstaats als seines Herkunftsstaats teilzunehmen und daraus Nutzen zu ziehen, wodurch die wirtschaftliche und soziale Verflechtung innerhalb der Gemeinschaft im Bereich der selbständigen Tätigkeiten gefördert wird ". Vergleich in diesem Sinne Rechtssache 2/74 Urteil des Gerichtshofes vom 21. Juni 1974, Reyners, Slg. 1974, S. 631, Rdn. 21.

28 Rechtssache 75-63 Urteil des Gerichtshofes vom 19. März 1964, Hoekstra (née Unger), Slg. 1964, S. 381, Rdn. 177. 
leistet. Dabei musste geklärt werden, ob der Arbeitnehmerbegriff national oder gemeinschaftsrechtlich auszulegen ist. Die Kommission war der Auffassung, dass der Begriff Arbeitnehmer im Sinne der Richtlinie 77/187/EEG auf Gemeinschaftsebene zu bestimmen sei. Laut der Kommission erfasse die Richtlinie jeden, der gegen Bezahlung eine Arbeit für Rechnung eines anderen verrichte, demgegenüber er sich in einem Unterordnungsverhältnis befinde. Da die Richtlinie nur eine teilweise Harmonisierung auf dem betreffenden Gebiet vornimmt, indem sie hauptsächlich den den Arbeitnehmern durch die Rechtsvorschriften den Mitgliedstaaten selbst bereits gewährten Schutz auch auf den Fall des Unternehmensübergangs ausdehnt, können sich dementsprechend auch nur diejenigen auf die Richtlinie berufen, die auf die eine oder andere Weise nach den Rechtsvorschriften des betreffenden Mitgliedstaats als Arbeitnehmer geschützt sind, urteilte jedoch der EuGH. Der Begriff Arbeitnehmer im Sinne dieser Richtlinie ist demnach so zu verstehen, ,, dass er alle Personen erfasst, die in dem betreffenden Mitgliedstaat als Arbeitnehmer aufgrund der nationalen arbeitsrechtlichen Vorschriften geschützt sind" 29

Ebenfalls hier einschlägig ist die Rechtssache Wippel. ${ }^{30}$ Aus ihr geht, kurz gesagt, hervor, dass auch Arbeitnehmer mit einem Teilzeitarbeitsvertrag, in dem die Wochenarbeitszeit und die Ausgestaltung der Arbeitszeit nicht festgelegt sind, sondern sich nach den von Fall zu Fall bestimmten Erfordernissen des Arbeitsanfalls richten und die Teilzeitbeschäftigten zwischen der Annahme und der Ablehnung dieser Arbeit wählen können, in dem Anwendungsbereich der Richtlinie 97/81/EG ${ }^{31}$ fallen.

\section{Definition des Arbeitnehmerbegriffes durch die Gemeinschaft}

Die skizzierte Situation führt schon seit geraumer Zeit zu der Frage, ob die EG nicht einen Arbeitnehmerbegriff einführen sollte, dessen Geltungsbereich auch die innerstaatliche Anwendung erfasst. Die arbeitsrechtlichen Richtlinien geben Anlass zur Frage, welche Reichweite sie eigentlich haben, d.h. welche Personen sich auf diese Richtlinien berufen können und welche nicht. Das folgt aus der skizzierten Begriffsbestimmung der Richtlinien. Zum einen findet man in den englischsprachigen Richtlinien die Begriffe „employee“ und ,worker“. ${ }^{32}$ Weshalb im englischen verschiedene Arbeitnehmerbegriffe gebraucht werden, ist nicht klar. Auch kann keine einheitliche Linie in dem Gebrauch dieser beiden Begriffe gesehen werden. Zum anderen werden die Begriffe

29 Rechtssache 105/84 Urteil des Gerichtshofes vom 11. Juli 1985, Foreningen af Arbejdsledere i Danmark gegen A/S Danmols Inventar (Mikkelsen), Slg. 1985, S. 2639, Rdn. 22-28.

30 Rechtssache C-313/02 Urteil des Gerichtshofes vom 12. Oktober 2004, Wippel, Slg. 2004, Seite I-9483.

31 Richtlinie 97/81/EG des Rates vom 15. Dezember 1997 zu der von UNICE, CEEP und EGB geschlossenen Rahmenvereinigung über Teilzeitarbeit - Anhang: Rahmenvereinbarung über Teilzeitarbeit, Amtsblatt Nr. L 14 vom 20.1.1998, S. 9-14.

32 Z.B. Richtlinien die den Begriff employee verwenden: 91/533/EG, 94/45/EG, 2001/23/EG, 2001/86/EG und 2002/14/EG. Z.B. Richtlinien die den Begriff worker verwenden: 96/71/EG und 98/59/EG. Richtlinie 2003/88/EG verweist sowohl auf employees als auch auf workers. 
Arbeitsvertrag und Arbeitsverhältnisse ${ }^{33}$ mal zusammen in Richtlinien genannt, mal auch nur der erste Begriff. Es scheint, als würde der Begriff „worker“" gebraucht, um anzudeuten, dass es nicht nur um Stammarbeitnehmer mit unbefristetem Arbeitsvertrag geht, sondern auch um atypische Arbeitsverhältnisse. Was ein Arbeitsverhältnis ist, wird in den Richtlinien nicht definiert.

Dass einige Richtlinien sich auf Arbeitnehmer mit unbefristetem Arbeitsvertrag als auch Arbeitnehmer in anderen Arbeitsformen beziehen, deutet unmissverständlich auf eine Erweiterung des Anwendungsbereiches der Richtlinien hin. Für reguläre Arbeitnehmer, die aufgrund eines eindeutigen Arbeitsvertrages arbeiten, ist das unproblematisch. Schwierig wird die Bestimmung des Anwendungsbereiches jedoch bei Personen, die nicht eindeutig Arbeitnehmer bzw. Selbständige sind. Gerade für diese ,Zwischengruppen" bedarf aber oftmals eines besonderen Schutzes. Zudem ist der Unterschied zwischen Unselbständigen und Selbständigen nicht mehr klar, weshalb die Bestimmung beider Kategorien in den einzelnen Mitgliedstaaten weit auseinander fallen können, je nach dem, wie sich die Rechtsprechung in dem betreffenden Staat entwickelt.

In seinem Bericht über die erneuerte Sozialagenda der Europäischen Kommission, bedauert das Europäische Parlament, dass die Kommission in ihrer Sozialagenda keinen Vorschlag gemacht hat ,, [...] eine Richtlinie zu grundlegenden Arbeitsrechten für alle Arbeitnehmer ungeachtet ihrer arbeitsrechtlichen Lage zum Schutze der wachsenden Zahl atypischer Arbeitsverhältnisse", einzuführen. ${ }^{34}$

Die Meinungen, ob es einen gemeinschaftsweiten einheitlichen Arbeitnehmerbegriff geben sollte, der dazu dient, den Unterschied zwischen Unselbständigen und Selbständigen klarer zu definieren, gehen auseinander. Das lässt sich unter anderem den Kommentaren derjenigen entnehmen, die sich an der öffentlichen Anhörung des Grünbuches über die Modernisierung des Arbeitsrechts beteiligt haben. Auch die Wissenschaft ist geteilter Auffassung. ${ }^{35}$ Nun soll zuerst auf das Grünbuch der Kommission eingegangen werden, um im Anschluss die IAO-Empfehlung 198 und deren Bedeutung kurz zu erläutern. Bevor wir zu unserer Analyse kommen, schlagen wir eine mögliche Arbeitnehmerdefinition vor, wie sie eventuell in der EG Anwendung finden könnte.

33 Richtlinien die sowohl Arbeitsvertrag als auch Arbeitsverhältnis verwenden: 91/533/EEG (Pflicht des Arbeitgebers zur Unterrichtung des Arbeitnehmers über die für seinen Arbeitsvertrag oder sein Arbeitsverhältnis geltenden Bedingungen), 97/81/EG (Rahmenvereinigung über Teilzeitarbeit), 1999/70/EG (Rahmenvereinbarung über befristete Arbeitsverträge) und 2008/104/EG (Leiharbeit).

34 Europäisches Parlament, Bericht über die erneuerte Sozialagenda (2008/2330(INI), A6-0241/2009, 3. April 2009, Absatz 6.

35 Rebhahn (Fn. 10) S. $154 \mathrm{ff}$ und in der Fortsetzung R. Rebhahn, Arbeitnehmerähnliche Rechtsvergleich und Regelungsperspektive, RdA 4/2009, S. 236-253. Ebenfalls zu dem Thema: Perulli (Fn. 1) S. 107 und Supiot (Fn. 3). Siehe auch B. Grandi, 'Would Europe Benefit from the Adoption of a Comprehensive Definition of the Term 'Employee' Applicable in all Relevant Legislative Modes?', International Journal of Comparative Labour Law and Industrial Relations 2008, Vol. 24/4, p. 495-510, worin die Autorin die (möglichen) Folgen der Einführung eines gemeinschaftsrechtlichen Arbeitnehmerbegriffes untersucht. 


\section{a) Das Grünbuch}

In dem Grünbuch der Kommission aus 2006 mit dem Titel „Ein modernes Arbeitsrecht für die Herausforderungen des 21. Jahrhunderts“ wird festgestellt, dass die herkömmliche Unterscheidung zwischen abhängigen Beschäftigten und Selbständigen nicht mehr angemessen die wirtschaftlichen und sozialen Gegebenheiten der Arbeitswelt spiegelt. Demzufolge wird in dem Grünbuch die Frage formuliert, ob die in den Mitgliedstaten geltenden Definitionen von Beschäftigung und Selbständigkeit nicht eine größere Klarheit erfordern. Ebenfalls wurde die Frage gestellt, ob es nicht eines Grundstocks an Vorschriften bedarf, welche die Beschäftigungsbedingungen aller Beschäftigten, unabhängig von der Form des Vertrags, regeln. ${ }^{36}$ Es ist davon auszugehen, dass hiermit alle unselbständig Beschäftigten gemeint sind.

In dem Grünbuch wird argumentiert, dass Mindestanforderungen für alle persönlichen Dienstleistungsverträge von wirtschaftlich abhängigen Selbständigen, eingeführt werden sollten. Trotz der Tatsache, dass so die Sicherheit und Transparenz erhöht und einen Mindestschutz gewährleist werden könnte, besteht andererseits die Gefahr, dass ein solcher Kriterienkatalog die Anwendung der Vertragsvereinbarungen - in concreto die Diensleistungs- und Niederlassungsfreiheit - einschränken. ${ }^{37}$

Dem Europäischen Parlament zufolge, liegt es im Interesse einer effizienteren Anwendung des Gemeinschaftsrechts, zu einer Angleichung der einzelstaatlichen Arbeitnehmerdefinitionen zu kommen. ${ }^{38}$ Dennoch ist sich das Parlament bewusst, dass es aufgrund der beträchtlichen Unterschiede bei den sozialen und wirtschaftlichen Gegebenheiten und der verschiedenen Traditionen der Mitgliedstaaten sehr kompliziert, ist eine einheitliche Arbeitnehmerdefinition einzuführen.

Die Arbeitgebervertreter sowie auch einige Mitgliedstaaten verteidigen die Meinung, dass die Definition der Arbeitnehmereigenschaft in den arbeitsrechtlichen Richtlinien weiterhin im Ermessen der Mitgliedstaaten liegen soll. Leider lassen sie Begründungen vermissen, weshalb sie gegen die Einführung eines gemeinschaftsweiten Arbeitnehmerbegriffs sind. ${ }^{39}$ Interessenvertreter der Sozialpartner der Dienstleistungs-, Unterhaltungs-, Medien- und Einzelhandelsbranche meinen hingegen, dass es sinnvoll sein könnte, wenigstens die in den Mitgliedstaaten verwendeten Definitionen für Personen, die als Freiberufler, Gelegenheitsarbeiter oder Selbständige bezeichnet werden, ,aufzulisten und zu erläutern, um ein besseres Verständnis der arbeitsrechtlichen Stellung dieser Personen zu ermöglichen " ${ }^{40}$

Anstelle eines gemeinschaftsrechtlichen Arbeitnehmerbegriffes, haben wiederum andere vorgeschlagen, dass die Kommission Leitlinien zur Beurteilung der Selbständigkeit

36 Europäische Kommission, Grünbuch, Ein modernes Arbeitsrecht für die Herausforderungen des 21. Jahrhunderts, $\operatorname{KOM}(2006) 708$ endgültig, S. 12-14.

37 KOM(2006) 708 endgültig (Fn. 36) S. 14. Siehe ebenfalls Rebhahn (Fn. 35) S. 246 ff.

$38 \operatorname{KOM}(2007) 627$ endgültig (Fn. 15) S. 8.

39 Business Europe meint sogar, dass die einzelstaatlichen Definitionen eines Arbeitnehmers klar und deutlich formuliert sind und aufgrund dessen keine Vereinheitlichung auf gemeinschaftsrechtlicher Ebene notwendig sei. Siehe Position Paper Business Europe, Green Paper Modernising Labour Law to Meet the Challenges of the 21 st Century, vom 6. März 2007, S. 8.

$40 \operatorname{KOM}(2007) 627$ endgültig (Fn. 15) S. 8. 
oder Unselbständigkeit aufstellen sollte. ${ }^{41}$ Zudem forderte das Europäische Parlament die Mitgliedstaaten auf, die Durchsetzung der IAO-Empfehlung 198 zu fördern. ${ }^{42}$

\section{b) Die IAO-Empfehlung 198 betreffend das Arbeitsverhältnis}

Nicht nur die EG, sondern auch die IAO hat sich mit den Problemen und Schwierigkeiten bzgl. des Arbeitsverhältnisses auseinandergesetzt. Die IAO „ist der Auffassung, dass es schwierig ist, das Vorliegen eines Arbeitsverhältnisses festzustellen, wenn die jeweiligen Rechte und Pflichten der betreffenden Parteien nicht klar sind, wenn versucht worden ist, das Arbeitsverhältnis zu verschleiern, oder wenn die Gesetzgebung, ihre Auslegung oder ihre Anwendung Unzulänglichkeiten oder Grenzen aufweisen “. Von einem verschleierten Arbeitsverhältnis kann, so die Empfehlung, gesprochen werden, wenn ein Arbeitgeber eine Person anders als einen Arbeitnehmer auf eine Weise behandelt, die deren wahren rechtlichen Status als Arbeitnehmer verdeckt, wodurch Situationen entstehen können, in denen vertragliche Regelungen dazu führen, dass Arbeitnehmern der ihnen zustehende Schutz vorenthalten wird. Demnach sollten die Mitgliedstaaten bestimmte Maßnahmen ergreifen, welche die genannten Probleme zur Feststellung eines Arbeitsvertrages beheben oder begrenzen. Ob ein Arbeitsvertrag vorliegt, sollte auf der Grundlage von Tatsachen bestimmt werden. Dabei kommt es nicht darauf an, wie die Rechtsbeziehung in einer vertraglichen oder anderen Vereinbarung charakterisiert wird.

Bezüglich der Indikatoren, die bei der Beurteilung, ob ein Arbeitsverhältnis vorliegt, eine wichtige Rolle spielen, schlägt die IAO vor, dass die Mitgliedstaaten die folgenden Indikatoren in ihren Rechtsvorschriften festlegen sollten. Erstens die Tatsache, dass die Arbeit nach den Weisungen und unter der Kontrolle einer anderen Person verrichtet wird; dass sie die Integration des Beschäftigten in die Organisation des Unternehmens mit sich bringt; dass Arbeit ausschließlich oder hauptsächlich für eine andere Person verrichtet wird; dass sie von dem Beschäftigten persönlich geleistet werden muss; dass sie innerhalb eines bestimmten Zeitrahmens oder an dem von der Person, die die Arbeit verlangt, angegebenen oder vereinbarten Arbeitsplatz ausgeführt wird; dass die Arbeit mit einer bestimmten Dauer verbunden ist und eine bestimmte Kontinuität aufweist; dass sich der Beschäftigte zur Verfügung hält oder dass die Werkzeuge, Materialien und Maschinen von der Person, die die Arbeit verlangt, gestellt werden. Und zweitens die regelmäßige Zahlung des Entgelts des Beschäftigten; die Tatsache, dass ein solches Entgelt die einzige oder die Haupteinnahmequelle des Beschäftigten darstellt; Sachbezüge wie Nahrungsmittel, Wohnung oder Transport; die Anerkennung von Ansprüchen wie wöchentliche Ruhezeit und Jahresurlaub; die Bezahlung der berufsbedingten Fahrten des Beschäftigten durch die Person, die die Arbeit verlangt; oder das Fehlen finanzieller Risiken für den Beschäftigten.

Die genannten Indikatoren sind in den (meisten) Mitgliedstaaten der EU Grundlage für die Charakterisierung eines Arbeitsverhältnisses. Jedoch muss man dabei bedenken,

41 Unter anderem Belgien, Deutschland, Irland, Italien und Lettland.

42 ILO-Empfehlung 198 betreffend das Arbeitsverhältnis, angenommen auf der 95. Tagung der internationalen Arbeitskonferenz im Juni 2006. Eine Zusammenfassung der ILO-Empfehlung findet man in Engels (Fn. 1) S. $339 \mathrm{ff}$. 
dass bestimmte Indikatoren in dem einen Mitgliedstaat eine wichtigere Rolle spielt als in dem anderen.

\section{c) Ein möglicher gemeinschaftsweiter Arbeitnehmerbegriff?}

Die Diskussion bezüglich eines gemeinschaftsweiten Arbeitnehmerbegriffes wird dadurch erschwert, dass die Autoren, die sich mit diesem Thema auseinandersetzen, bislang keinen möglichen gemeinschaftsweiten Arbeitnehmerbegriff definiert haben. Die Frage, was ein solcher Begriff beinhalten soll, wird meistens außer Acht gelassen. Demnach kann die Frage in den Raum gestellt werden, ob überhaupt derselbe Arbeitnehmerbegriff gemeint ist. Bevor ein einheitlicher Arbeitnehmerbegriff in zwischenstaatlichen Sachverhalten Anwendung finden kann, sollte erst einmal überlegt werden, ob eine solche Definition zunächst nur für Richtlinien eingeführt werden sollte. Sollte sich diese Strategie als erfolgreich erweisen, besteht die Möglichkeit, dann den Anwendungsbereich dieser Definition zu erweitern. Dabei geht es auch darum zu bestimmen, inwieweit es den Mitgliedstaaten gestattet ist, selbst Kriterien zur weiteren Ausarbeitung und Deutung des gemeinschaftsrechtlichen Arbeitnehmerbegriffes einzuführen.

Die gemeinschaftlichen Rechtspraktiken der Mitgliedstaaten, die IAO-Empfehlung 198 und die Rechtsprechung des EuGHs im Fall Lawrie-Blum können als mögliche Grundlage des gemeinschaftsrechtlichen Arbeitnehmerkonzeptes dienen. ${ }^{43}$ Eine mögliche Definition, basierend auf der Rechtssache Lawrie-Blum, könnte wie folgt aussehen: „Von einem Arbeitsverhältnis kann gesprochen werden, wenn jemand während einer bestimmten Zeit für einen anderen nach dessen Weisung Leistungen erbringt, für die er als Gegenleistung eine Vergütung erhält.“ Diese Definition umfasst nicht nur jene Personen die aufgrund eines Arbeitsvertrages arbeiten, sondern auch solche Personen, die aufgrund eines sonstigen Arbeitsverhältnisses Leistungen erbringen. ${ }^{44}$

Bezüglich der Frage ob eine Unterordnung vorhanden ist, können die Mitgliedstaaten die folgenden Tests handhaben. Erstens die „,persönliche Unterordnung“. Dieses Kriterium dient dazu zu beurteilen, ob eine Person unter der Kontrolle/Unterordnung einer anderen Person dieser Person Anweisungen geben kann als auch wie, wann und wo die Arbeit verrichtet werden soll. In diesem Kontext können die Mitgliedstaaten ebenfalls in Betracht ziehen, ob eine Person Teil einer Organisation und/oder Produktion ausmacht. Zweitens die ,wirtschaftliche Abhängigkeit“. Damit soll angedeutet werden, ob eine Person regulär für eine andere Person arbeitet und/oder die Bezahlung ausschließlich durch diese Person erhält. Drittens die ,rechtliche Abhängigkeit“". Mit diesem Kriterium kann beurteilt werden, ob eine Person einer anderen Person untergeordnet ist und die letzte Person Anweisungen geben kann.

Zusätzlich sollte den Mitgliedstaaten die Möglichkeit gegeben werden, andere Indikatoren festzustellen, die verdeutlichen, ob ein Arbeitsvertrag oder ein Arbeitsverhältnis

43 Auch Perulli (Fn. 1) S. 15 und 16 nennt ähnliche Kriterien zur Bestimmung der unselbständigen und selbständigen Erwerbstätigkeit.

44 Es ist darauf hinzuweisen, dass das European Labour Law Network in Kürze ein Restatement herausgeben wird über das Thema Arbeitsverhältnisse in den Mitgliedstaaten der EU. Für Informationen zum Netzwerk, siehe http://www.labourlawnetwork.eu. 
existiert. Die Mitgliedstaaten können den folgenden Indikatoren Bedeutung beimessen: Die betreffende Person: arbeitet hauptsächlich für einen Auftraggeber und/oder wird davon abgehalten, ähnliche Arbeit für eine andere Person zu verrichten; verrichtet ausschließlich Arbeit; kann die Arbeit nicht mittels eines Untervertrags weiterleiten; arbeitet eine gewisse Anzahl an Stunden pro Woche; erhält einen Stunden-, Wochen- oder Monatslohn; beschafft nicht die Betriebsmittel sowie auch die Arbeitsmaterialien für die Arbeit; bekommt seine Ausgaben erstattet; tritt nicht selbständig auf dem Markt auf; arbeitet nicht auf eigene Rechnung; profitiert nicht von der Verrichtung der Arbeit; trägt nicht die typischen unternehmerischen Risiken, kann diese nicht beeinflussen und befindet sich in einer ähnlichen wirtschaftlichen bzw. sozialen Situation wie gewöhnliche Arbeitnehmer.

Eine solche Arbeitnehmerdefinition in Verbindung mit den skizzierten Kriterien und Indikatoren lässt den Mitgliedstaaten genügend Entscheidungsfreiheit für die Beurteilung, ob eine Person als Arbeitnehmer klassifiziert werden soll oder nicht. Eine solche Entscheidungsfreiheit ist notwendig, damit nicht jene Personen ausgeschlossen werden, die nicht aufgrund eines Arbeitsvertrages arbeiten, sich jedoch in einer arbeitnehmerähnlichen Position befinden. Bezüglich arbeitnehmerähnlicher Personen könnte in den Richtlinien ausdrücklich gesagt werden, dass diese ebenfalls in den Anwendungsbereich der betreffenden Richtlinie fallen bzw. dass sie explizit vom Anwendungsbereich der Richtlinien ausgeschlossen werden.

\section{Die Einführung einer Zwischengruppe}

Im Prinzip stützen sich die Mitgliedstaaten auf ein binäres Modell, das zwischen Beschäftigungsverhältnissen und beruflicher Selbständigkeit differenziert. Zwischenformen der Beschäftigung sind in vielen Mitgliedstaaten nicht gesetzlich verankert. Allerdings gibt es zwischen einem „Beschäftigungsverhältnis” und der „Selbständigkeit” durchaus eine Zwischenkategorie, auch als „Grauzone” bezeichnet werden kann. Personen, die dieser Kategorie zuzuordnen sind, haben einen undeutlichen Status und besitzen im Rahmen des Arbeitsrechts nur einige Sonderrechte, obwohl ihre Situation in gewisser Hinsicht der eines Arbeitnehmers ähnelt. Personen, die in diese Zwischenkategorie einzuordnen sind, lassen sich als wirtschaftlich abhängige Personen beschreiben, da sie einen unabhängigen Status im Hinblick auf ihre vertragliche Arbeitsbeziehung mit einem Kunden oder einem Unternehmen besitzen. In der Tat handelt es sich dabei um juristisch unabhängige, jedoch wirtschaftlich abhängige Personen. Die IAO beschreibt wirtschaftlich abhängige Personen in ihrem Bericht wie folgt: ,, Midway between the employment relationship and self-employment, there are 'economically dependent workers' who are formally self-employed but depend on one or a few 'clients' for their income. They are not easy to describe, let alone quantify, because of the heterogeneous nature of the situations involved and the lack of a definition or statistical tool." 45

45 ILO Report V(1), The employment relationship, International Labour Conference, $95^{\text {th }}$ Session 2003, S. 12. 
In einigen Ländern sind ,wirtschaftlich abhängige Beschäftigte” vom Gesetz als solche anerkannt (Deutschland, Italien und Spanien). Andere Länder besitzen keine juristische Definition, obwohl diese Kategorie in der Praxis durchaus vorkommt oder auch verwendet bzw. diskutiert wird (z.B. Dänemark, Finnland, Frankreich, Griechenland, Irland, die Niederlande, Norwegen, Österreich, Portugal und die Tschechische Republik). In Ländern, wo solche Beschäftigte offiziell anerkannt werden, gelangen mehrere verschiedene Konzepte zum Einsatz. Zudem werden dieser Kategorie unterschiedliche Rechte zugeschrieben.

In Spanien hat der Gesetzgeber eine spezifische Kategorie von wirtschaftlich abhängigen Arbeitern gesetzlich eingeführt. Die wesentlichen Merkmale dieser Kategorie bestehen darin, dass diese Personen eine professionelle Tätigkeit für nur einen Kunden ausüben, wofür sie als Gegenleistung eine entsprechende Entlohnung erhalten. Sie sind vom Kunden wirtschaftlich abhängig, wenn sie mindestens 75 Prozent des gesamten Einkommens von diesem Kunden beziehen. Um als wirtschaftlich abhängig eingestuft zu werden, darf ein Beschäftigter keine Arbeitnehmer einstellen, und er darf die fragliche Tätigkeit nicht weiter vergeben. Auch darf er keine Dienstleistungen in genau der gleichen Weise wie die Arbeitnehmer des Kunden erbringen. Zudem muss er oder sie seine eigenen Strukturen, Betriebsmittel und Materialien besitzen; muss eine Entlohnung in Abhängigkeit von den erzielten Ergebnissen erhalten und seine eigenen finanziellen Risiken tragen Auch darf er oder sie die fragliche Tätigkeit nicht auf Körperschaftsbasis ausüben. Beschäftigte, die in diesem Sinn als ,wirtschaftlich abhängige Personen“ klassifiziert werden, genießen teilweise den Schutz, den auch „Arbeitnehmer" in Anspruch nehmen können.

In Deutschland gibt es so genannte arbeitnehmerähnliche Personen, die in mancher Hinsicht einen ähnlichen Status besitzen wie Arbeitnehmer, da es sich hierbei um ,wirtschaftlich abhängige" Personen handelt, die teilweise auch den für Arbeitnehmer geltenden Rechtsschutz in Anspruch nehmen können. Sie haben Anspruch auf Jahresurlaub, unterliegen dem Gleichbehandlungsprinzip, und eventuelle Rechtsstreitigkeiten, in die sie involviert sind, können an ein Arbeitsgericht verwiesen werden. Einige Merkmale arbeitnehmerähnlicher Personen sind in $\S 12$ a des Tarifvertragsgesetzes festgelegt: die wirtschaftliche Abhängigkeit (im Gegensatz zur persönlichen Abhängigkeit oder der Unterordnung); ${ }^{46}$ der Bedarf an sozialen Schutz aufgrund von erbrachter und noch zu erbringen Dienstleistungen, die persönlich und ohne die Hilfe hierfür eingestellter Arbeitnehmer durchgeführt werden; ${ }^{47}$ und weil entweder die Arbeit im Wesentlichen für eine Person erledigt wird oder die erwerbstätige Person mehr als die Hälfte des Gesamteinkommens von einer einzigen Firma bezieht. ${ }^{48} 49$

In Italien gibt es ebenfalls gesetzlich anerkannte ,wirtschaftlich abhängige Selbständige" oder auch quasi-subordinierte Beschäftigte. Diese Gruppe besteht im Wesentlichen aus kooperativen Beziehungen, die im Zusammenhang mit einer „laufenden, ununterbrochenen Erbringung von Dienstleistungen” bzw. „Projektarbeit” zum Tragen kommen können, wobei es sich um eine laufende, ununterbrochene Erbringung von

$46 \S 12$ a Tarifvertragsgesetz.

$47 \S 12$ a Tarifvertragsgesetz.

$48 \S 12$ a Tarifvertragsgesetz und $\S 7$ Sozialgesetzbuch, Viertes Buch.

49 Siehe auch Perulli (Fn. 1) S. 86 und 89. 
Dienstleistungen im Zusammenhang mit einem spezifischen Projekt handelt. Das italienische Gesetz definiert solche Arbeitsvereinbarungen auch als ,,versteckte Arbeit”, und zwar aufgrund der Tatsache, dass die Abgrenzung zwischen Arbeitnehmern und selbständig erwerbstätigen Personen nach Auffassung des Gesetzgebers in gewisser Hinsicht obsolet geworden ist. Der Rechtsschutz für diese Personengruppe beinhaltet auch soziale Sicherheit, beispielsweise mit Altersruhegeld, Schutz vor Betriebsunfällen und Krankheiten, Mutterschutz und Krankengeld. ${ }^{50}$

Als weiteres Beispiel sei Großbritannien erwähnt. Dort hat man, wenn auch ohne Verwendung des Begriffes arbeitnehmerähnliche Personen bzw. Selbständige, die Kategorie des „worker“ eingeführt. Diese Kategorie umfasst Personen die weder Arbeitnehmer („,employee“) noch Selbständige (,self-employed“) sind. ${ }^{51}$ Mit dem Begriff „worker" bezeichnet man eine breitere Kategorie, die Arbeitnehmer aber auch die, die sich vertraglich verpflichtet haben, persönlich eine Arbeit oder eine Dienstleistung für eine andere Partei zu erbringen, und deren vertragsmäßiger Status nicht der eines Benutzers bzw. eines Abnehmers der von der Gegenpartei ausgeübten gewerblichen oder kaufmännischen Tätigkeit ist. ${ }^{52}$ Der britische Gesetzgeber hat das Diskriminierungsverbot, den Mindestlohn, die Arbeitszeitregelung und die Rechte der Teilzeitarbeitnehmer auf die ,worker“" ausgedehnt. ${ }^{53}$

Die Kategorie der so genannten ,,arbeitnehmerähnlichen Personen” gibt es auch, unter anderem, in Österreich, den Niederlanden und Portugal. Die relevanten gesetzlichen Vorgaben beziehen sich auf Personen, die Arbeiten verrichten oder Dienstleistungen erbringen, die von und im Auftrag einer anderen Person ohne Arbeitsvertrag und ohne juristische Unterordnung aufgetragen worden sind. In Österreich beziehen sich nur wenige Vorschriften des Arbeitsrechts auf arbeitnehmerähnliche Personen. Beispielsweise sind Vorschriften bezüglich der Arbeitsgerichte, der Tätigkeit im Auftrag von Zeitarbeitsunternehmen, der Haftung der Arbeitnehmer und der Anti-Diskriminierungsgesetze auf diese Gruppe anwendbar. Andere Rechte werden von den Gerichten zugesprochen, sofern sie keine ,persönliche Unterordnung” erfordern. Somit beziehen sich gewisse Teile des Arbeitsrechts (z.B. Kündigungsschutz, bezahlter Urlaub, Lohnfortzahlung bei Krankheit) nicht auf arbeitnehmerähnliche Personen. In Portugal beziehen sich ebenfalls gewisse Vorschriften des Arbeitsrechts auf wirtschaftlich abhängige erwerbstätige Personen, nämlich die juristischen Vorgaben in Bezug auf die Persönlichkeitsrechte, Gleichberechtigung und Gleichstellung, Arbeitssicherheit und Gesundheit. ${ }^{54}$ Wirtschaftlich abhängige Arbeiter gibt es zudem auch im Rechtssystem/Arbeitsrecht der Niederlande. Die außerordentliche Beschäftigungsverordnung, die auch das Kündigungsrecht reguliert, gilt auch in Fällen, wo kein Arbeitsvertrag vorliegt, wo jedoch die fragliche Person persönliche Arbeiten im Auftrag einer anderen Person ausführt, sofern er oder sie nicht für zwei oder mehr Personen arbeitet, zwei oder mehr Personen

50 Diese Rechte sind zu finden in: Artikel 2113 Zivilgesetzbuch, Artikel 409 Zivilprozessordnung, Artikel 2 Absatz 36-31 Gesetz Nr. 335 von 1995, Artikel 5 rechtsvertretendes Dekret Nr. 38 von 2000 und Artikel 59 Gesetz Nr. 447 von 1997.

51 Bzgl. der Kategorie der ,worker“ in Großbritannien siehe G. Davidov, Who is a Worker?, Industrial Law Journal 2005, Vol. 34/1, S. 57-71.

52 Industrial Relations Act 1996.

53 Siehe auch Perulli (Fn. 1) S. 89 bis 91.

54 Artikel 10 des portugiesischen Arbeitsgesetzbuches. 
zur Unterstützung einstellt und auch kein Ehepartner, kein eingetragener Partner oder ortsansässiger Blutsverwandter oder angeheirateter Verwandter ist, oder wenn es sich hierbei nicht um sekundäre Arbeit handelt. Vergleichbare Definitionen gibt es in der niederländischen Sozialgesetzgebung.

\section{Analyse}

In diesem Abschnitt gehen wir der Frage nach, ob eine einheitliche europäische Arbeitnehmerdefinition eingeführt werden soll bzw. gewünscht ist. Ebenfalls besprechen wir die potenzielle Einführung einer dritten Kategorie, die sich zwischen den regulären Arbeitnehmern und den Selbständigen befinden. Es ist nicht unser Anliegen, eine allumfassende Analyse vorzulegen. Unser Vorschlag ist lediglich als Anregung für weitere Gedankengänge und Überlegungen gedacht.

\section{Europäische Arbeitnehmerdefinition}

Eine europäische Arbeitnehmerdefinition, die zunächst nur in den arbeitsrechtlichen Richtlinien Anwendung findet, könnte dazu beitragen, die nationalen Definitionen zu ersetzen und umzuformulieren. Um den Schutz der Arbeitnehmer auf atypische Arbeitsverhältnisse ausbreiten zu können, bedarf es einer solchen Neudefinition bzw. Ausweitung des Arbeitnehmerbegriffs. ${ }^{55}$ Eine solche Ausweitung findet man, wie oben schon angedeutet, in einigen Richtlinien, deren Anwendungsbereich nicht nur jene Personen erfasst, die einen klassischen Arbeitsvertrag haben, sondern auch solche, die sich in anderen Formen eines Beschäftigungsverhältnisses befinden. Dadurch könnte die Verbreitung der so genannten Scheinselbständigkeit verhindert und die eigentliche Selbständigkeit verbessert werden. Ebenfalls hätte das zur Folge, dass der gegenwärtige Schutz der gemeinschaftsrechtlichen Richtlinien, automatisch ausgeweitet werden würde auf andere abhängig Beschäftigte. Das hieße, dass nicht nur Grundrechte zugesprochen würden, sondern auch Rechte, die in Richtlinien und anderen Gemeinschaftsvorschriften aufgenommen sind.

Laut dem Perulli-Bericht wird diese Auffassung jedoch nur von einer Minderheit vertreten. Grund dafür ist - wahrscheinlich - die übermäßige Ausweitung des Begriffs der unselbständigen Arbeit und der entsprechenden Schutzvorschriften auf gegenwärtig noch als selbständig klassifizierte Personen. Vor allem ,, der Mangel an Selektivität und die übermäßige Gleichstellung des arbeitnehmerähnlichen Selbständigen mit dem Arbeitnehmer" spricht dagegen. ${ }^{56}$ Eine Ausdehnung könnte, laut Rebhahn, auch nur dann verwirklicht werden, wenn das Schutzniveau deutlich reduziert würde. ${ }^{57}$

Ein weiteres Hindernis, das gegen die Einführung spricht, wird darin gesehen, dass durch die Einführung eines gemeinschaftsweiten Arbeitnehmerbegriffes die Mitglied-

55 Grandi (Fn. 36) S. 502 und 504. Die Ausbreitung bestimmter Teile des Arbeitsrechts auf die Personen die weder Arbeitnehmer noch Arbeitgeber sind wird auch bestätigt durch den $\mathrm{Su}$ piot Bericht. Siehe Supiot (Fn. 3) S. 218-220.

56 Perulli (Fn. 1) S. 122.

57 Rebhahn (Fn. 35) S. 245. 
staaten nicht mehr genügend Freiraum für weitere Differenzierungen haben würden. Eine verstärkte Differenzierung sollte aber weiterhin durch die Mitgliedstaaten vorgenommen werden können. ${ }^{58}$ Dieser Einwand bedarf der Relativierung. Dass ein gemeinschaftsweiter einheitlicher Arbeitnehmerbegriff zwingend bedeutet, dass die Mitgliedstaaten nicht mehr befugt sind, Differenzierungen vorzunehmen, ist keineswegs zwingend. Bevor eine solche Schlussfolgerung gezogen werden kann, muss schließlich erst festgestellt werden, wie denn ein gemeinschaftsrechtlicher Arbeitnehmerbegriff aussehen könnte und was dessen Reichweite ist. Geht man davon aus, dass dieser Begriff sehr weit ausgelegt wird, kann angenommen werden, dass es den Mitgliedstaaten nicht mehr - oder nur sehr beschränkt - erlaubt ist, weitere Differenzierungen vorzunehmen. Würde man aber eher einen sehr eng auszulegenden Begriff einführen und es den Mitgliedstaaten überlassen, diesen Begriff auf andere abhängig Beschäftigte auszudehnen, spräche im Prinzip nichts gegen die Einführung eines gemeinschaftsweiten Arbeitnehmerbegriffes.

Es sollte in diesem Zusammenhang geklärt werden wie sich eine (widerlegliche) Vermutung zugunsten des gemeinschaftsrechtlichen Arbeitnehmerbegriffes zu der Dienstleistungs- und Niederlassungsfreiheit in grenzüberschreitenden Situationen verhält Für Personen, deren Status undeutlich ist, haben einige Gesetzgeber in den Mitgliedstaaten Rechtsvermutungen zu Gunsten der Arbeitnehmereigenschaft bzw. der Selbständigkeit kreiert. Oftmals sind diese Vermutungen widerlegbar. ${ }^{59}$ Eine Vermutungsregel bestimmt, wie bei Zweifel an einer bestimmten Situation, aber auch unabhängig davon, zu entscheiden ist. Im Prinzip kann eine widerlegliche Vermutung den Arbeitnehmerbegriff de facto erweitern. ${ }^{60}$ In den Niederlanden z.B. gibt es seit 1999 eine widerlegliche Vermutung für das Vorliegen eines Arbeitsvertrages. Diese Vermutung besagt, dass wenn jemand länger als drei Monate regelmäßig mindestens zwanzig Stunden im Monat gegen Bezahlung Dienste erbringt, für diese Person ein Arbeitsvertrag angenommen wird. ${ }^{61}$ Ein potenzielles Hindernis für eine gesetzlich geregelte Vermutungsregel könnten die Dienstleistungs- und Niederlassungsfreiheit sein (Artikel 39 und 43 EGV).

Wenn eine Person in dem einen Staat als selbständig klassifiziert wird, jedoch in dem anderen Staat aufgrund einer Vermutung als Arbeitnehmer einzustufen ist, dann bedeutet dies, dass diese Vermutung die Dienstleistungsfreiheit beschränkt oder gar zur Seite schiebt. In der Rechtssache Kommission/Frankreich geht der EuGH davon aus, dass: „Im vorliegenden Fall stellt die Vermutung der Arbeitnehmereigenschaft unabhängig davon, wie schwer sie zu widerlegen ist, eine Behinderung des freien Dienstleistungsverkehrs im Sinne von Artikel 49 EG dar. Denn auch wenn sie den fraglichen Künstlern nicht eigentlich die Möglichkeit nimmt, ihre Tätigkeit in Frankreich als Selbständige

58 Rebhahn (Fn. 35) S. 252.

59 Eine widerlegliche Rechtsvermutung gibt es z.B. in den Niederlanden. Diese besagt, dass ein Arbeitsvertrag vorliegt, wenn eine Person gegen Bezahlung zumindest während drei aufeinanderfolgende Monate für mindestens 20 Stunden in der Woche für eine andere Person arbeitet (Artikel 7:610 a Niederländisches Bürgerliches Gesetzbuch). In Italien findet man eine ähnliche Rechtsvermutung. Frankreich z.B. hat eine Rechtsvermutung gegen die Arbeitnehmereigenschaft.

60 Rebhahn (Fn. 10) S. 171.

61 Artikel 7:610 a des Niederländischen Bürgerlichen Gesetzbuches. Auch in Spanien und Frankreich gibt es Vermutungsregeln. 
auszuüben, so beschwert sie sie doch in einer Weise, die geeignet ist, ihre Tätigkeit als Dienstleister zu behindern. " Artikel 49 EGV verlangt nicht nur die Beseitigung jeder Diskriminierung von Dienstleistenden aus anderen Mitgliedstaaten, sondern auch die Abschaffung aller Beschränkungen der Dienstleistungsfreiheit. Dabei spielt keine Rolle, dass diese ohne Unterschied auch für inländische Dienstleistende wie für solche aus anderen Mitgliedstaaten gelten. Solang diese Unterschiede dazu geeignet sind die Tätigkeiten von Dienstleistenden aus den anderen Mitgliedstaaten, die in ihrem Herkunftsmitgliedstaat rechtmäßig ähnliche Dienstleistungen erbringen, zu unterbinden, zu behindern oder weniger attraktiv zu machen. ${ }^{62}$ Bei der soeben genannten Rechtssache ist zu betrachten, dass diese Vermutung nur jene Arbeitenden betraf, die einen künstlerischen Beruf ausgeübt haben und in ihrem Herkunftsmitgliedstaat als Dienstleister anerkannt sind und dort in der Regel ähnliche Dienstleistungen erbringen. ${ }^{63}$ Eine widerlegliche Vermutung wird eher akzeptiert, wenn sie eine große Gruppe an Personen betrifft, als wenn sie nur eine kleine Anzahl an Personen davon erfasst wird. ${ }^{64}$

Wie wir sehen können Qualifikationsprobleme in grenzüberschreitenden Situationen dann auftreten, wenn eine Vermutungsregel zugunsten der Arbeitnehmereigenschaft entscheidet. Diese Probleme könnten dadurch behoben werden, dass Personen, die in ihrem Herkunftsmitgliedstaat als Selbständige tätig sind, ebenfalls als solche in anderen Mitgliedstaaten klassifiziert werden. Man könnte für grenzüberschreitende Situationen eine Art Vermutung kreieren, die besagt, dass die Qualifizierung im Herkunftsmitgliedstaat im anderen Mitgliedstaat bestehen bleibt.

Eine andere Lösung könnte folgendes Beispiel sein. In den Niederlanden gibt es für Selbständige eine Möglichkeit, vom Finanzamt eine Erklärung - eine so genannte Verklaring Arbeidsrelatie - zu bekommen, die beinhaltet, dass diese Person für die Dauer eines Kalenderjahres als selbständig klassifiziert wird. Diese Erklärung hat zur Folge, dass, so lange diese Erklärung gültig ist, für den Richter als auch für das Finanzamt und sonstige Behörden die für Rentenansprüche und soziale Sicherheit zuständig sind, die Selbständigkeit der betreffenden Person feststeht. Diese Erklärung kann nicht widerlegt werden. Eine solche Erklärung könnte auch innerhalb der EG in grenzüberschreitenden Fällen als Beweis dafür anerkannt werden, dass die betreffende Person selbständig ist. $^{65}$

\section{Einführung einer Zwischenkategorie}

Die (gesetzliche) Einführung oder der Ausbau einer solchen Zwischenkategorie hat, unter anderem, den Vorteil, dass man gewisse Schutzaspekte besser differenzieren kann.

62 Rechtssache C-76/90 Urteil vom 25. Juli 1991, Säger, Slg. 1991, I-4221, Rdn. 12; Rechtssachen C-49/98, C-50/98, C-52/98, C-54/98, C-68/98 und C-71/98 Urteil vom 25. Oktober 2001, Finalarte u. a., Slg. 2001, I-7831, Rdn. 28.

63 Rechtssache 255/04 Urteil des Gerichtshofes vom 15. Juni 2006, Kommission/Frankreich, Slg. 2006, S. 5251, Rdn. 36-38.

64 Rebhahn (Fn. 35) S. 247.

65 Erst kürzlich hat ein niederländisches Gericht geurteilt, dass eine Belgierin die in den Niederlanden als Krankenpflegerin tätig ist, eine solche Erklärung beantragen kann die als Beweis dient für Auftraggeber und mögliche Sozialabgaben. Siehe Rechtbank Breda 22. Juli 2009, AWB 09/673, LJN BJ4743. 
Eine Zwischenkategorie mit einem Arbeitnehmer gleichzustellen und dieser Kategorie den gleichen Schutz bzw. die gleichen Rechte zu geben, scheint nicht das Richtige zu sein. Dann könnte man den Unterschied zwischen Selbständigkeit und Arbeitnehmereigenschaft gleich einebnen. Das würde die (hohe) Schutzbedürftigkeit des Arbeitnehmers und damit dessen Sonderstellung verkennen. Eine selektive Ausdehnung des Schutzes auf Zwischenkategorien dürfte deshalb die beste Lösung sein.

Bei der Gruppe der abhängig Beschäftigten geht es um Personen, die keine direkten Anweisungen von ihrem Auftraggeber erhalten, von dem sie (hauptsächlich) abhängig sind. Dabei könnte das Kriterium ,während einer bestimmten Zeit", das bereits erwähnt wurde, eine entscheidende Rolle spielen. Je länger diese Person sich in einem abhängigen Beschäftigungsverhältnis mit seinem Auftraggeber befindet, umso eher kann angenommen werden, dass es sich um ein Arbeitsverhältnis handelt.

Bislang gehören die wirtschaftlich abhängigen Beschäftigen zu der (Unter)Kategorie der Selbständigen. Aus den oben genannten Länderbeispielen geht nicht hervor, dass diese einen gesonderten Vertragstypus initiiert haben. Um wirtschaftlich abhängige Beschäftigte leichter von Selbständigen bzw. Arbeitnehmern unterscheiden zu können, ist zu überlegen, ob nicht ein gesonderter Vertragstypus erarbeitet werden sollte. ${ }^{66}$

Aus dem Perulli-Bericht ergibt sich, dass ein nationaler Gesetzgeber prinzipiell zwei Strategien verfolgt: zum einen die partielle Gleichstellung und zum anderen die selektive Ausdehnung der Arbeitnehmereigenschaft auf wirtschaftlich abhängige Beschäftigte. Deutschland und Italien können in Bezug auf die partielle Gleichstellung genannt werden, d.h. dass es nur bezüglich einiger Teilbereiche eine Gleichstellung gibt. Was die selektive Ausdehnung angeht, so gibt es in Großbritannien zwei verschiedene Kriterien der selektiven Ausdehnung. Der erste betrifft die Einführung des „worker“ und der zweite betrifft die Befugnis der Regierung, Erwerbstätigen, auf die die Gesetze nicht anwendbar sind, verschiedene Rechte zu gewähren. ${ }^{67}$

Dem Perulli-Bericht ist zu entnehmen, dass die zweite Variante nicht unproblematisch ist, weil sie tendenziell die Aushöhlung des Schutzes der unselbständigen Arbeit zur Folge haben kann. Die Kategorie der wirtschaftlich abhängigen Beschäftigten sollte demzufolge weiterhin als (Unter)Kategorie der Selbständigen betrachtet werden. ${ }^{68}$

\section{Ein Grundstock an Rechten für alle abhängigen Beschäftigten?}

Schließlich wäre zu diskutieren, ob es erstrebenswert ist, dass die gemeinschaftsrechtliche oder nationale Gesetzgebung ein Grundstock an Rechten für alle abhängig Beschäftigte vorgibt, ungeachtet des jeweiligen Status in Bezug auf das Beschäftigungsverhältnis. Dadurch könnte der Missbrauch der im Regelfall schwächeren Position dieser berufstätigen Personen vermieden werden, weil ihnen einen gewisser Mindeststandard in Bezug auf eine angemessene Arbeit garantiert würde. Dabei stellt sich dann natürlich die Frage, welcher Mindeststandard hier gelten sollte. Dieser Vorschlag findet

66 Siehe auch Rebhahn (Fn. 35) S. $248 \mathrm{ff}$.

67 Perulli (Fn. 1) S. $115 \mathrm{ff}$.

68 Perulli (Fn. 1) S. 124. 
sich ebenfalls wieder im Perulli-Bericht, wo darauf verwiesen wird, dass er in der europäischen Rechtslehre immer mehr Zustimmung findet. ${ }^{69}$

Auch im Supiot-Bericht wird einen Grundstock an Rechten für alle abhängigen Beschäftigten vorgeschlagen, wobei zwischen vier so genannten Kreisen differenziert wird: (1) allgemeingültige Sozialrechte, die ungeachtet der ausgeübten Tätigkeit garantiert werden; (2) Rechte, die auf nichtberuflichen Arbeit basieren; (3) Rechte, die teilweise im Gemeinschaftsrecht aufgenommen worden sind und die für alle Berufe gelten, wie z.B. Gesundheitsschutz und Sicherheit; und (4) das individuelle Arbeitsrecht. Das Gleichbehandlungsprinzip gilt für alle genannten Kreise. Sowohl der Supiot- als auch der Perulli-Bericht befürworten die Einführung ,,sozialer Grundrechte für alle Beschäftigungsverhältnisse“. Dieses Konzept scheint ein geeigneter Weg zu sein, der Forderung nach ,, active security in uncertain circumstances " gerecht zu werden. ${ }^{70}$

Widmet man sich der Frage, welche Rechte genau den Grundstock bilden sollten, so könnten beispielsweise Rechte in Bezug auf Mindestlöhne, Urlaubsansprüche, Arbeitszeit oder auch die Sozialgesetzgebung zu diesem Grundstock gehören.

\section{Schlussfolgerung}

Es gibt einen gemeinschaftsrechtlichen Arbeitnehmerbegriff im Hinblick auf die Freizügigkeit der Arbeitnehmer (Artikel 39 EGV). Dieser Begriff ist sehr global und bringt deshalb nur wenige Probleme mit sich. In Bezug auf Richtlinien gibt es jedoch (noch) keinen gemeinschaftsrechtlichen Arbeitnehmerbegriff, so dass bei Grenzfällen fraglich ist, ob die Richtlinie überhaupt Anwendung finden kann. Um zu einem einheitlichen Anwendungsbereich zu gelangen, könnte man einen einheitlichen Arbeitnehmerbegriff entwickeln. In diesem Artikel haben wir anzudeuten versucht, wie eine solche Vereinheitlichung aussehen könnte.

Die Argumente gegen einen gemeinschaftsrechtlichen Arbeitnehmerbegriff, soweit es sich um politische Argumente handelt, gründen hauptsächlich in der Befürchtung, dem Arbeitsrecht könnten weitere nationale Befugnisse entzogen werden. Das ist insoweit richtig, als der EuGH die Rechtsprechung auf Gemeinschaftsniveau weiterentwickelt und demnach von nationalen Rechtsvorstellungen abweichen kann.

Dass dadurch die Konkurrenz auf diesem Rechtsgebiet vermindert werden könnte, wird allerdings in dieser Argumentation unterbewertet. Die Bedenken gegen einen gemeinschaftsrechtlichen Arbeitnehmbegriff wären wahrscheinlich geringer, würde man sich erst einmal mit dem Inhalt eines solchen Begriffes beschäftigen. Vielleicht haben die Mitgliedstaaten mehr gemeinsam, als sie je gedacht haben. Dann erscheint die Einführung eines solchen Begriffes alles andere als abwegig. Der erste Schritt müsste deshalb darin bestehen, die Gemeinsamkeiten herauszuarbeiten.

Auch im Hinblick auf die Einführung einer dritten Gattung, nämlich wirtschaftlich abhängige Beschäftigte, wird befürchtet, diese könnte die Rechte der Arbeitnehmer gefährden. Dazu kommt die Angst, man könnte dieser Kategorie bestimmte Ansprüche bzw. Rechte zuerkennen, die sie möglicherweise gar nicht haben möchte. Auch hier

69 Perulli (Fn. 1) S. 122.

70 Supiot (Fn. 3) S. 222. 
wäre erst zu überlegen, welche Rechte dieser Gruppe zugesprochen werden sollten bzw. welche Rechte zugesprochen werden sollen. Erst wenn über konkrete Vorschläge gesprochen wird, kann beurteilt werden, ob ein solches Vorgehen in der Praxis erwünscht und umsetzbar ist. Dabei ist wichtig, dass ein weiterer Vertragstypus entwickelt werden sollte, der sich deutlich vom Arbeits- bzw. Dienstleistungsvertrag unterscheidet.

Schließlich haben wir die Frage aufgeworfen, ob alle abhängig Beschäftigten mit einem Grundstock an Rechten ausgestattet werden sollen, unabhängig von der Art und Beschreibung ihrer Beschäftigung. Dieser Lösungsvorschlag scheint, momentan jedenfalls, am ehesten umsetzbar zu sein. Jedoch sollte auch hier erst einmal genau geklärt werden, welche Rechte für alle abhängig Beschäftigten und welche Rechte nur für bestimmte Gruppen von Beschäftigten garantiert werden sollten. Überdies bedarf die Einstufung als „abhängig Beschäftigter“ noch der Klärung. 